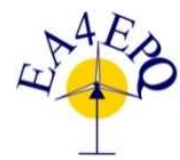

International Conference on Renewable Energies and Power Quality (ICREPQ'15)

La Coruña (Spain), $25^{\text {th }}$ to $27^{\text {th }}$ March, 2015

Reneurable Energy and Pourer Quality. Tournal (RE\&PQJ)

ISSN 2172-038 X, No.13, April 2015

\title{
Characterization of current switching transients for appliance identification
}

\author{
Yulieth Jimenez ${ }^{1}$, Cesar Duarte ${ }^{1}$, Johann Petit ${ }^{1}$, Jan Meyer $^{2}$, Peter Schegner ${ }^{2}$, Gilberto Carrillo ${ }^{3}$ \\ ${ }^{1}$ School of Electrical, Electronics and Telecommunications Engineering. \\ Universidad Industrial de Santander - UIS \\ Bucaramanga (Colombia) \\ e-mail: yulieth.jimenez@correo.uis.edu.co, cedagua@uis.edu.co, jfpetit@uis.edu.co \\ ${ }^{2}$ Institute of Electrical Power Systems and High Voltage Engineering. \\ Technical Universitaet Dresden - TUD \\ Dresden (Germany) \\ e-mail: jan.meyer@tu-dresden.de, peter.schegner@tu-dresden.de \\ ${ }^{3}$ Universidad de Santander - UDES. \\ Bucaramanga (Colombia) \\ e-mail: gilberto.carrillo@udes.edu.co
}

\begin{abstract}
Demand Side Management is a key concept within the Smart Grid vision to promote energy efficiency, load flexibility and interaction between the consumers and other power grid stakeholders. Disaggregated information requires an advanced load monitoring system of individual appliance consumption. A smart house is envisioned to include a Nonintrusive Load Monitoring (NILM) system to support demand side management and motivate the users to adopt energy saving practices. NILM systems use input electrical measurements taken at the energy meter point of a house and estimate the individual appliance operation and consumption through mathematical algorithms. Each appliance can be distinguished from others through a set of particular attributes namely load signatures that can be computed from transient signals, steady state signals or both. This paper aims to characterize current switching transients, for NILM applications and to discuss how they are affected by variation of factors such as point on wave of switching, network impedance, supply voltage distortion and sampling frequency of the meter. For that purpose, measurements of residential appliances of several categories are acquired and processed. The conclusion of this work is the assessment of suitability, robustness and efficiency of appliance identification based on current transients.
\end{abstract}

\section{Key words}

Smart grids, nonintrusive load monitoring, current switching transients, appliance identification.

\section{Introduction}

The fast electricity demand growth and the possibility of shortages impelled the search of alternatives to supply and even reduce this demand [1]. The increase of supply capacities requires high investments in new generation and brings environmental damage for the scarcity of the natural resources and the greenhouse gas emissions, especially for the fossil fuel based generation. The high energy losses during the generation, transmission and distribution process actually announce that too many units of primary energy are lost in order to carry one single electricity unit to the final user. This scenario keeps the worldwide community interested not only in expediting the penetration of renewable energy sources but also in formulating good thermal building design and reducing the demand peaks.

Studies indicate that reductions of between $10 \%$ and $25 \%$ in electricity demand are achievable through the deployment of Smart Grids [1]. The control of power usage at the consumer side, namely Demand Side Management, is a key concept within the Smart Grid vision. This feature promotes energy efficiency, load flexibility and interaction between the consumers and other power grid stakeholders, through strategies such as demand response (direct load control or voluntary pricebased load shifting), energy saving practices or energy efficient technologies [2]. These strategies require advanced load monitoring, not only to know the user consumption per month but also detailed and disaggregated information about the individual appliance consumption and usage behavior.

Traditionally, load monitoring consists on using either historical information, that leads to inaccurate estimations, or systems with one dedicated sensor per appliance, which are more accurate but increase hardware efforts and costs in installation and maintenance of sensors and communication platforms. In order to overcome these drawbacks, another research field has become important recently: Nonintrusive Load Monitoring (NILM). One approach of these applications is to use electrical measurements taken at the house energy meter point and estimate the individual appliance 
operation and consumption through mathematical algorithms [3].

A smart house is envisioned to include a NILM system to provide more detailed and frequent feedback than the monthly electricity bill, for example, to conform an adaptive load priority list (non-interruptible devices) for each consumer. Moreover, studies demonstrate that this feedback motivates the users to modify their consumption behaviors resulting in savings of up to $15 \%$ [4]. So even if the disaggregated information were not used by further Smart Grid applications, NILM would have important benefits for the customers.

Each appliance might be distinguished from others through a set of particular attributes exhibited in the electrical signals. This discriminant information, namely load signatures, can help to identify the appliances. Load signatures can be computed from transient signals (e.g. switching of the appliances), steady state signals (e.g. during the operation of the appliance) or both [5]. This paper focuses on the transient state signals, during switching an appliance on. Although previous works have considered transients, some questions remain still open. The reproducibility of the switching transients for a single appliance is important for the identification process, but can be considerably affected by impact factors such as point on wave of switching (point on the voltage wave where the appliance is switched), network impedance, supply voltage distortion and sampling frequency of the metering equipment. No comprehensive research results are known to the authors in those issues. In order to analyze the impact of the aforementioned factors, measurements of several categories of residential appliances are acquired and processed.

The paper is organized as follows. Section 2 presents some background about the appliances selected for the study. Afterwards, Section 3 discusses the measurement framework and section 4 introduces the indices, which are used to characterize the transients in both time and frequency domain. Section 5 presents the results of the measurements, while section 6 summarizes the results in a conclusion.

\section{Categorization of household appliances}

The behavior of switching transients is expected to differ significantly between different types of devices. Electronic and non-electronic devices have to be distinguished. Non-electronic equipment includes e.g. resistive heating or directly connected machines. Electronic loads can be distinguished by the power factor correction (PFC) implementation [6].

No PFC: These topologies consist of a rectifier and the DC link capacitor without any additional PFC components. Examples are Compact Fluorescent Lamps (CFL) with rated power below $25 \mathrm{~W}$ and some chargers of electronic devices.

Passive PFC: These circuits add passive components such as capacitors or inductors before or after the rectification stage, as low frequency filters, in order to diminish the current distortion. Examples are selected power supplies for desktop PC and selected LED lamps.

Active PFC: These topologies add active components like DC-DC converters and achieve the lowest distortion and the best power factor. Examples are some CFLs with rated power above $25 \mathrm{~W}$ for $230 \mathrm{~V} / 50 \mathrm{~Hz}$ and also some power supplies for desktop PC.

\section{Measurement framework}

\section{A. Experimental Set-up}

Fig. 1 shows details about the experimental set-up to measure the switching transients in the laboratory. The equipment under test (EUT) is connected to a power amplifier that simulates the $120 \mathrm{~V} / 60 \mathrm{~Hz}$ supply. For a more realistic operation, the appliances are switched manually instead of using an electronic switch (sometimes the equipment includes its own switch). An A/D converter connected to a computer controls the amplifier. Voltage and current measurements are taken simultaneously at a sampling frequency of $2 \mathrm{MHz}$ and stored at a computer where the data are processed off line.

IEC 60725 [7] provides typical values of the network impedance $Z$ in public low voltage grids with $230 \mathrm{~V} / 50 \mathrm{~Hz}$ supply. Based on this impedance a value for $120 \mathrm{~V} / 60 \mathrm{~Hz}$ was derived with $\mathrm{Z}=(0.6+j 0.075) \Omega$. This impedance is shown as dotted box in Fig. 1.

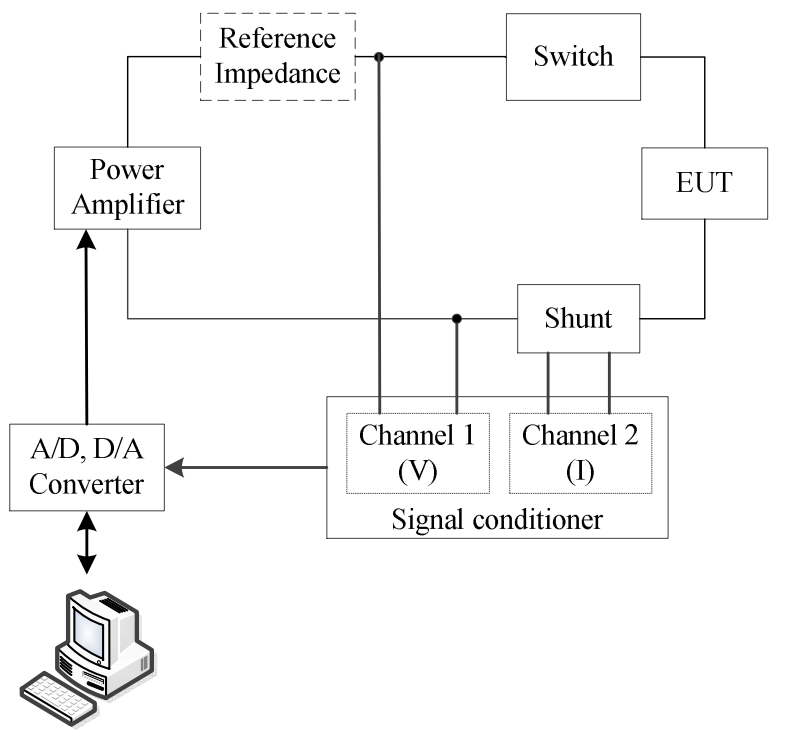

Fig. 1. Measurement Set-up

\section{B. Appliance selection}

This study involves non-electronic and electronic equipment. The categories of electronic devices described in Section 2 were also included. Table I shows the list of the seven EUT used in the laboratory along with their power ratings. Fig. 2 shows the waveforms of the currents carried by the appliances under a nondistorted $120 \mathrm{~V} / 60 \mathrm{~Hz}$ voltage supply. 
Table I. - Equipment under test

\begin{tabular}{|c|c|c|c|}
\hline \multicolumn{2}{|c|}{ Category } & Appliance & $\begin{array}{c}\text { Rated } \\
\text { Power[W] }\end{array}$ \\
\hline \multirow{2}{*}{$\begin{array}{l}\text { Non- } \\
\text { electronic }\end{array}$} & Resistive & Halogen Lamp & $53 \mathrm{~W}$ \\
\hline & Resistive & Hair Dryer & $1875 \mathrm{~W}$ \\
\hline \multirow{5}{*}{ Electronic } & \multirow{2}{*}{ No PFC } & $\mathrm{CFL}$ & $18 \mathrm{~W}$ \\
\hline & & Laptop charger & $50 \mathrm{~W}$ \\
\hline & $\begin{array}{c}\text { Passive } \\
\text { PFC }\end{array}$ & LED lamp & $1.2 \mathrm{~W}$ \\
\hline & \multirow{2}{*}{$\begin{array}{l}\text { Active } \\
\text { PFC }\end{array}$} & PC power supply 1 & 400W \\
\hline & & PC power supply 2 & 430W \\
\hline
\end{tabular}

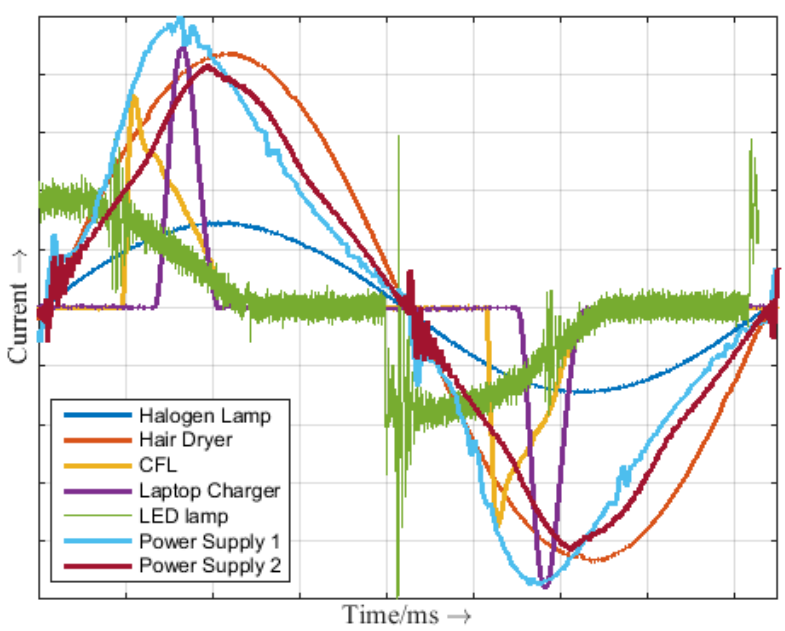

Fig. 2. Characteristic waveforms of the currents drawn by the appliances under a non-distorted $120 \mathrm{~V} / 60 \mathrm{~Hz}$ voltage supply for a fundamental period.

\section{Measurement procedure}

Switching measurements are carried out with only one appliance at the same time. Each appliance is randomly switched hundred times in order to ensure a good coverage of the half wave by the switching moment between $0^{\circ}$ and $180^{\circ}$. The voltages are used to calculate these angles. The currents to identify begin and end of the transient as well as to perform the characterization for appliance identification.

\section{Indices for characterization of current switching transient}

Once the measurements are acquired, the samples are processed off-line in MATLAB ${ }^{\circledR}$. The signals are segmented and filtered as a preprocessing step. Next they are characterized by a set of indices in the time domain. In addition, the data is transformed to frequency domain and a further set of indices is computed.

\section{A. Time domain}

Be $\mathbf{x}=\left[x_{1} x_{2} \ldots x_{N}\right]$ a series of discrete values that represent the samples of the current $x(t)$. Statistical measures were computed according to the following equations:
Crest

factor

$$
c f=\frac{|\mathbf{x}|_{\max }}{|\mathbf{x}|_{\text {rms }}}=\frac{|\mathbf{x}|_{\text {max }}}{\sqrt{\frac{1}{N} \sum_{i=1}^{N}|x|_{i}^{2}}}
$$

Power

$$
p=\mathbf{x}_{r m s}^{2}=\frac{1}{N} \sum_{i=1}^{N}|x|_{i}^{2}
$$

Standard deviation

$$
\sigma=\sqrt{\frac{1}{N} \sum_{i=1}^{N}\left(x_{i}-\overline{\mathbf{x}}\right)^{2}}
$$

where $\overline{\mathbf{x}}=\frac{\mathbf{1}}{N} \sum_{i=1}^{N} x_{i}$ is the mean of $\mathbf{x}$.

$$
\begin{array}{ll}
\text { Skewness } & \gamma=\frac{\frac{1}{N} \sum_{i=1}^{N}\left(x_{i}-\overline{\mathbf{x}}\right)^{3}}{\sigma^{3}} \\
\text { Kurtosis } & k=\frac{\frac{1}{N} \sum_{i=1}^{N}\left(x_{i}-\overline{\mathbf{x}}\right)^{4}}{\sigma^{4}} \\
\text { Entropy } & \mathcal{E}=-\sum_{i=1}^{N} \mathbf{x} \cdot \log _{2} \mathbf{x}
\end{array}
$$

Crest factor indicates how pointed is the signal with respect to the RMS value. Power, similar to standard deviation, accounts to the variability or dispersion including the mean. Skewness and kurtosis are measures of the shape. Finally, entropy measures the randomness of the switching transient. The duration, $\boldsymbol{N}$, of the switching transient was also considered as an index.

\section{B. Frequency domain}

A switching transient is a non-stationary signal because its frequency content changes with time. In this sense, transformations, e.g. S-transform, are more useful than classical spectral analysis tools to characterize the signals. The continuous S-transform, $S(\tau, f)$, of a signal $x(t)$ is the convolution between the signal $x(t)$ multiplied by an exponential $e^{-j 2 \pi f t}$ and a Gaussian window function $g_{f}(t)[8]$ :

$$
S(\tau, f)=\int_{-\infty}^{\infty} x(t) e^{-j 2 \pi f t} g(t-\tau) d t
$$

where $g_{f}(t)=\frac{|f|}{\sqrt{2 \pi}} e^{-\frac{t^{2} f^{2}}{2}}$.

This transform can be calculated for discrete signals as well. The algorithm to compute this transform is based on the Fast Fourier Transform. More details about the discrete S-transform, the algorithm implementation and the inverse S-transform can be found in [8].

The S-transform of a discrete signal results in a complex matrix that can be decomposed into a magnitude $\boldsymbol{A}$ and a phase $\varnothing$ over the time-frequency plane. Rows represent discrete frequencies and columns contribute with time location. Each column is like a local spectrum for that point in time. For the characterization of the switching transients, profiles are built by selecting the maxima per column of $\boldsymbol{A}$. 
Let $\mathbf{p}=\left[\begin{array}{llll}p_{1} p_{2} & \ldots & p_{M}\end{array}\right]$ be the frequency profile vector of the signal $\mathbf{x}$, where $p_{i}$ is the maximum of the $i^{\text {th }}$ column of $\boldsymbol{A}$. The indices computed from its frequency domain representation are: the energy, given by

$$
E=\frac{1}{M} \mathbf{p}_{r m s}^{2}=\frac{1}{M} \sum_{i=1}^{M}|\mathbf{p}|_{i}^{2},
$$

the standard deviation, the skewness, the kurtosis and the entropy of $\boldsymbol{p}$, which are computed analogously to the expressions for $\mathbf{x}$ in (3), (4), (5) and (6), respectively.

\section{Analysis of Impact Factors}

The time and frequency domain indices are used to assess the robustness of the identification in terms of impact factors that can occur under realistic conditions, namely the impact of switching moment, supply voltage distortion, supply impedance and sampling rate. Table II depicts the scenarios that are considered to analyze the effects of voltage distortion and supply impedance.

Table II. - Scenarios for the analysis of impact factors

\begin{tabular}{|c|c|c|c|}
\hline Scenario & $\begin{array}{c}\text { Supply } \\
\text { Voltage }\end{array}$ & Impedance $\mathbf{Z}$ & $\begin{array}{c}\text { No. } \\
\text { signals }\end{array}$ \\
\hline 1 & Sine & $0 \Omega$ & 604 \\
\hline 2 & Flat-top & $0 \Omega$ & 512 \\
\hline 3 & Sine & $0.6+j 0.075 \Omega$ & 739 \\
\hline 4 & Flat-top & $0.6+j 0.075 \Omega$ & 653 \\
\hline
\end{tabular}

\section{A. Point on wave of switching}

Switching transients might start at different instants of the momentary waveform, for example, close to the zero crossings of the waveform, or close to its minima or maxima. In general, this time instant affects the amplitude and the shape of the current switching transients, and even the frequency profiles. Fig. 3 and 4 show examples of the power (time domain) and the entropy of the switching transients (frequency domain) for all the appliances.

In general, all the switching transients show dependency on point on wave. Patterns occur for some of the indices, especially for the non-electronic appliances. On the contrary, the indices of the laptop charger behave more randomly and seem not to be influenced by the point on wave (Fig. 3 b and $4 b$ ).

The indices that show more correlation for the point on wave are:

- $\quad$ Time domain. Standard deviation and power, as can be presented in Fig. 3.

- Frequency domain. Standard deviation, energy and entropy, as presented in Fig. 4.

The duration is the only index that does not present any relationship with the point on wave. This is reasonable because the duration of the transient is directly related to the time constant which depends on the circuit elements. Table III and IV present a summary of the efficiency of the indices for the identification of the different appliances for Scenario 1. The values describe the linear separability of an index with respect to the same index of other appliances. To obtain those values all the combinations of pairs of appliances were evaluated to deduce the linear separability.

Table III.- Time domain indices for Scenario 1

\begin{tabular}{|c|r|c|c|c|c|c|}
\hline Appliance & $\boldsymbol{\sigma}, \boldsymbol{p}$ & $\boldsymbol{N}$ & $\boldsymbol{\varepsilon}$ & $\boldsymbol{\gamma}$ & $\boldsymbol{k}$ & $\boldsymbol{c f}$ \\
\hline Halogen Lamp & 4,0 & 3,8 & 1,0 & 1,9 & 1,9 & 0,0 \\
\hline Hair Dryer & 5,9 & 1,9 & 0,9 & 1,9 & 2,9 & 2,0 \\
\hline CFL & 2,9 & 3,0 & 1,0 & 5,5 & 5,7 & 3,7 \\
\hline Laptop Charger & 3,0 & 3,8 & 1,0 & 1,9 & 2,8 & 1,9 \\
\hline LED lamp & 4,0 & 6,0 & 4,9 & 6,0 & 6,0 & 4,0 \\
\hline Power Supply 1 & 5,0 & 1,0 & 0,0 & 1,9 & 2,0 & 1,9 \\
\hline Power Supply 2 & 5,0 & 1,9 & 1,0 & 1,9 & 1,9 & 1,9 \\
\hline
\end{tabular}

Table IV.- Frequency domain indices for Scenario 1

\begin{tabular}{|c|c|c|c|c|c|}
\hline Appliance & $\boldsymbol{\sigma}$ & $\boldsymbol{E}$ & $\boldsymbol{\varepsilon}$ & $\boldsymbol{\gamma}$ & $\boldsymbol{k}$ \\
\hline Halogen Lamp & 1,0 & 4,0 & 6,0 & 0,0 & 0,0 \\
\hline Hair Dryer & 0,9 & 2,0 & 3,9 & 2,0 & 2,0 \\
\hline CFL & 0,9 & 2,0 & 2,9 & 1,9 & 1,0 \\
\hline Laptop Charger & 1,9 & 5,9 & 4,6 & 0,0 & 0,0 \\
\hline LED Lamp & 2,0 & 5,9 & 3,9 & 1,9 & 1,0 \\
\hline Power Supply 1 & 1,0 & 3,0 & 3,0 & 0,0 & 0,0 \\
\hline Power Supply 2 & 3,9 & 3,0 & 4,9 & 1,8 & 0,0 \\
\hline
\end{tabular}

The linear separation is not $100 \%$ for few cases due to a quite small overlap in one or two measurements. In this case a weighting factor of 0.9 is applied to the values.

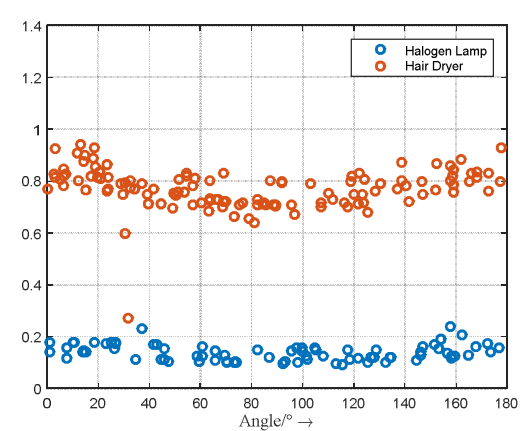

a)

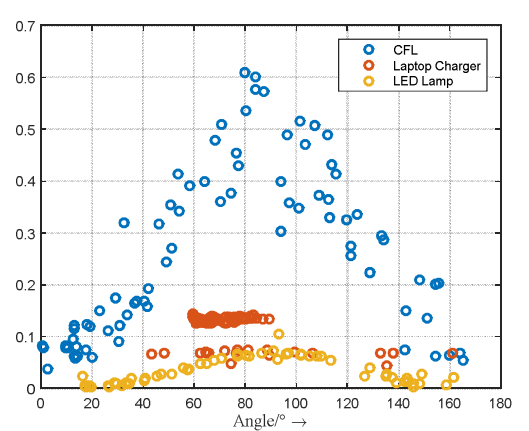

b)

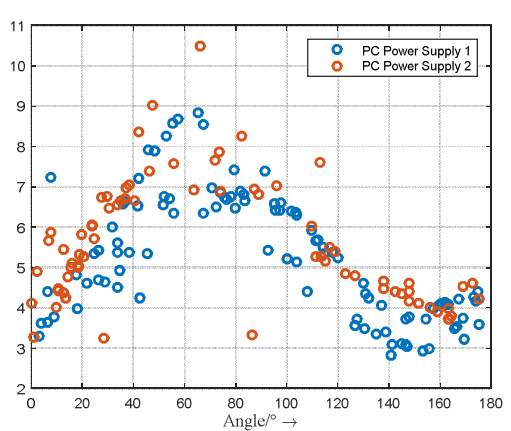

c)

Fig.3. Power of switching transients (time domain).

a) Non-electronic. b) Electronic: No PFC and Passive PFC. c) Electronic: Active PFC. 


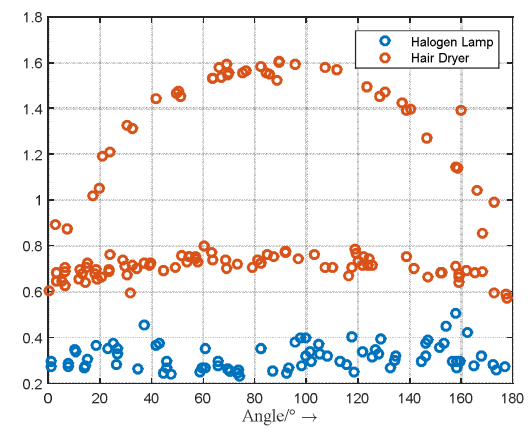

a)

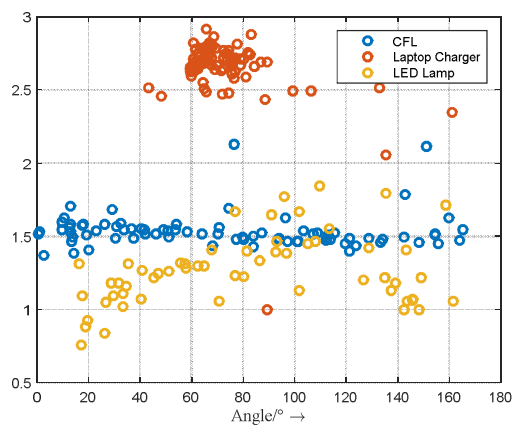

b)

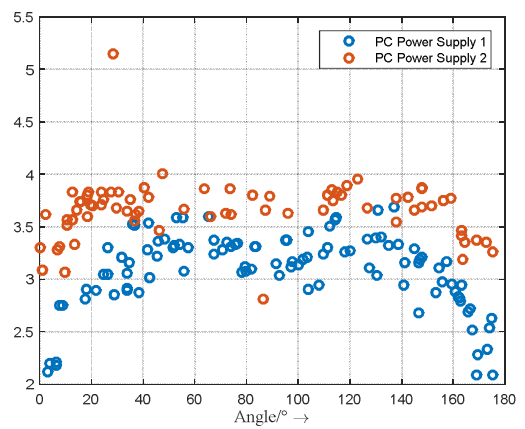

c)

Fig.4. Entropy of switching transients (frequency domain).

a) Non-electronic. b) Electronic: No PFC and Passive PFC. c) Electronic: Active PFC.

For example, consider the rows correspondent to the Halogen Lamp in both Tables. In Table III the value of 4,0 implies that the standard deviation and the power from time domain of the switching transients allow the distinction of the halogen lamp from other 4 appliances: the hair dryer (Fig. 3a), the LED lamp (Fig. 3b) and the PC power supplies (Fig. 3c). In Table IV, the entropy from frequency domain of the halogen lamp has a value of 6,0 because it is the only appliance with entropies below 0.5 , as is shown in Fig. 4, so it can be fully differentiated from the others. On the contrary, a value of zero would indicate overlap and the index would not be useful for appliance identification.

The suitability of indices for appliance identification is also identified by color. Red means low ability for separation while green represents a high suitability for appliance identification. The summation of all the values in Table III and IV indicates a total discrimination capacity of 198.8 for Scenario 1 (a total value of 462 would provide the ideal separability).

\section{B. Supply voltage distortion}

Generally residential LV grids show a flat-top voltage due to the mass use of single-phase rectifiers. Therefore the effect of a voltage distortion level on the switching transient is investigated. Table V and VI includes the indices for the flat-top voltage supply case and they are compared with the non-distorted voltage supply case in Tables III and IV.

Similar trends are observed for the indices in both Scenarios. For time domain the indices that provide the highest discriminant information are the standard deviation, the power and the kurtosis, while the entropy is not recommended for this purpose.

On the other hand, the frequency domain indices with more discriminant capacity are the energy and the entropy, while the standard deviation, the skewness and the kurtosis are not good indices for this purpose.

The summation of all the values is $\mathbf{1 9 0 . 2}$ for Scenario 2, which is less than the value of Scenario 1. In conclusion, the voltage distortion causes a small reduction in the ability of the indices to discriminate the appliances.
Table V.- Time domain indices for Scenario 2

\begin{tabular}{|c|c|c|c|c|c|c|}
\hline Appliance & $\boldsymbol{\sigma}, \boldsymbol{p}$ & $\boldsymbol{N}$ & $\boldsymbol{\varepsilon}$ & $\boldsymbol{\gamma}$ & $\boldsymbol{k}$ & $\boldsymbol{c f}$ \\
\hline Halogen Lamp & 4,0 & 5,0 & 0,0 & 1,9 & 2,9 & 1,9 \\
\hline Hair Dryer & 5,9 & 1,0 & 1,0 & 1,9 & 3,9 & 2,0 \\
\hline CFL & 3,9 & 3,0 & 1,0 & 3,6 & 3,7 & 3,9 \\
\hline Laptop Charger & 4,0 & 2,0 & 1,0 & 1,9 & 1,9 & 2,0 \\
\hline LED lamp & 6,0 & 5,0 & 5,0 & 5,0 & 5,0 & 4,0 \\
\hline Power Supply 1 & 5,0 & 2,0 & 1,0 & 1,9 & 1,9 & 1,0 \\
\hline Power Supply 2 & 5,0 & 2,0 & 1,0 & 1,0 & 1,9 & 1,0 \\
\hline
\end{tabular}

Table VI.- Frequency domain indices for Scenario 2

\begin{tabular}{|c|c|c|c|c|c|}
\hline Appliance & $\boldsymbol{\sigma}$ & $\boldsymbol{E}$ & $\boldsymbol{\varepsilon}$ & $\boldsymbol{\gamma}$ & $\boldsymbol{k}$ \\
\hline Halogen Lamp & 0,0 & 5,8 & 3,8 & 0,0 & 0,0 \\
\hline Hair Dryer & 0,0 & 4,9 & 4,9 & 2,0 & 2,0 \\
\hline CFL & 0,0 & 3,9 & 3,0 & 1,0 & 1,0 \\
\hline Laptop Charger & 0,0 & 5,9 & 4,9 & 0,9 & 0,9 \\
\hline LED lamp & 0,0 & 6,0 & 2,9 & 1,9 & 1,9 \\
\hline Power Supply 1 & 0,0 & 3,9 & 2,8 & 0,0 & 0,0 \\
\hline Power Supply 2 & 0,0 & 3,0 & 4,9 & 0,0 & 0,0 \\
\hline
\end{tabular}

\section{Network impedance}

Now the variation of the indices of the switching transients in the presence of supply impedance is assessed by comparing Tables VII and VIII with Tables III and IV.

For this scenario with supply impedance some trends can be identified in the indices too. For time domain the standard deviation, the power and the duration are the most discriminant indices, while the crest factor is the least discriminant one.

On the other hand, for frequency domain it is found that the energy and the entropy again have the best performance in the discrimination while the standard deviation, the skewness and the kurtosis have the worst one.

Table VII. - Time domain indices for Scenario 3.

\begin{tabular}{|c|r|c|c|c|c|c|}
\hline Appliance & $\boldsymbol{\sigma}, \boldsymbol{p}$ & $\boldsymbol{N}$ & $\boldsymbol{\varepsilon}$ & $\boldsymbol{\gamma}$ & $\boldsymbol{k}$ & $\boldsymbol{c f}$ \\
\hline Halogen Lamp & 5,0 & 3,0 & 1,0 & 0,0 & 0,0 & 0,0 \\
\hline Hair Dryer & 5,9 & 3,0 & 1,0 & 0,9 & 1,9 & 2,0 \\
\hline CFL & 3,9 & 1,0 & 1,0 & 0,0 & 0,0 & 0,0 \\
\hline Laptop Charger & 4,9 & 2,0 & 0,0 & 0,0 & 1,0 & 1,0 \\
\hline LED Lamp & 5,9 & 6,0 & 3,9 & 2,7 & 2,7 & 1,0 \\
\hline Power Supply 1 & 5,0 & 2,0 & 0,9 & 0,9 & 0,9 & 0,0 \\
\hline Power Supply 2 & 5,0 & 3,0 & 0,0 & 0,9 & 0,9 & 0,0 \\
\hline
\end{tabular}


Table VIII. - Frequency domain indices for Scenario 3.

\begin{tabular}{|c|c|c|c|c|c|}
\hline Appliance & $\boldsymbol{\sigma}$ & $\boldsymbol{E}$ & $\boldsymbol{\varepsilon}$ & $\boldsymbol{\gamma}$ & $\boldsymbol{k}$ \\
\hline Halogen Lamp & 0,0 & 5,0 & 2,9 & 0,0 & 0,0 \\
\hline Hair Dryer & 0,0 & 6,0 & 2,9 & 2,9 & 3,0 \\
\hline CFL & 0,0 & 4,0 & 5,9 & 2,0 & 2,0 \\
\hline Laptop Charger & 0,0 & 5,0 & 2,9 & 2,8 & 2,9 \\
\hline LED Lamp & 0,0 & 6,0 & 1,0 & 1,9 & 1,9 \\
\hline Power Supply 1 & 0,0 & 5,0 & 4,0 & 0,0 & 0,0 \\
\hline Power Supply 2 & 0,0 & 5,0 & 3,8 & 0,0 & 0,0 \\
\hline
\end{tabular}

The summation of all the values for the scenario with the reference impedance connected to the circuit is $\mathbf{1 5 9}$. Thus there is a meaningful reduction from the value of 198. 8 for Scenario 1 and it implies that the connection to a supply impedance attenuates the ability of the indices to distinguish the appliances.

\section{Sampling frequency}

The higher the sampling frequency the higher the details of switching transients, but also the necessary storage and processing power. In this case, measurements at $2 \mathrm{MHz}$ are downsampled to $40 \mathrm{kHz}$ to observe the effect of sampling frequency. Table IX and X reflect these results that are compared with Table VI and VII.

Table IX. - Time domain indices for Scenario 3 for $F s=$

\begin{tabular}{|c|c|c|c|c|c|c|}
\multicolumn{8}{c|}{$40 \mathrm{kz}$} \\
\hline Appliance & $\boldsymbol{\sigma}, \boldsymbol{p}$ & $\boldsymbol{N}$ & $\boldsymbol{\varepsilon}$ & $\boldsymbol{\gamma}$ & $\boldsymbol{k}$ & $\boldsymbol{c f}$ \\
\hline Halogen Lamp & 5,0 & 2,0 & 1,0 & 0,0 & 0,0 & 0,0 \\
\hline Hair Dryer & 6,0 & 2,0 & 1,0 & 0,9 & 0,9 & 2,0 \\
\hline CFL & 4,0 & 1,0 & 1,0 & 0,0 & 0,0 & 1,0 \\
\hline Laptop Charger & 4,9 & 3,0 & 0,0 & 0,0 & 0,0 & 0,0 \\
\hline LED Lamp & 5,9 & 6,0 & 3,9 & 0,9 & 0,9 & 1,9 \\
\hline Power Supply 1 & 5,0 & 2,0 & 0,9 & 0,0 & 0,0 & 0,9 \\
\hline Power Supply 2 & 5,0 & 2,0 & 0,0 & 0,0 & 0,0 & 0,0 \\
\hline
\end{tabular}

Table X. - Frequency domain indices for Scenario 3 for

\begin{tabular}{|c|c|c|c|c|c|}
\hline Fs $=40 \mathrm{kHz}$ \\
\hline Appliance & $\boldsymbol{\sigma}$ & $\boldsymbol{E}$ & $\boldsymbol{\varepsilon}$ & $\boldsymbol{\gamma}$ & $\boldsymbol{k}$ \\
\hline Halogen Lamp & 0,0 & 5,0 & 2,9 & 0,0 & 0,0 \\
\hline Hair Dryer & 0,0 & 6,0 & 2,9 & 2,9 & 3,0 \\
\hline CFL & 0,0 & 3,0 & 2,0 & 1,9 & 1,9 \\
\hline Laptop Charger & 0,0 & 5,0 & 0,0 & 1,8 & 1,9 \\
\hline LED Lamp & 0,0 & 5,0 & 0,0 & 1,0 & 1,0 \\
\hline Power Supply 1 & 0,0 & 5,0 & 2,0 & 0,0 & 0,0 \\
\hline Power Supply 2 & 0,0 & 5,0 & 1,8 & 0,0 & 0,0 \\
\hline
\end{tabular}

The trends of the index performance for appliance identification that were described in Subsection $5 \mathrm{~B}$ also apply for this case. However, the summation of all the values in the Tables is $\mathbf{1 3 2}$, which indicate a reduction of the ability of the indices to distinguish the different appliances.

\section{Conclusion}

Switching transients provide discriminative information about the individual type of appliance. Several indices can be computed for time and frequency domain. The most promising indices for appliances identification are:
1) Time domain. Standard deviation and power.

2) Frequency domain. Energy and entropy.

On the other hand, it is less recommended to use the standard deviation, the skewness and kurtosis of the frequency profiles.

Most of the indices of the current transients show dependency on the point on wave of switching, especially for the non-electronic appliances. The most independent index and appliance are the duration and the laptop charger, respectively.

The introduction of a supply impedance and a voltage distortion in the setup reduces the suitability of the indices for appliance identification. The presence of the supply impedance is the most critical impact factor. This should be considered for real applications, because certain impedance exists at any socket where an appliance can be connected. Subsequent the specific load signature of the appliance is harder to recognize.

The sampling frequency has also influence on the indices of the switching transients. According to the analysis, smaller sampling rates diminish the capacity of the indices for appliance identification. The sampling frequency should therefore be properly chosen in order to guarantee the appliance identification.

Under the different measurement conditions, the devices that exhibit more distinctive indices are the hair dryer and the LED lamp while the power supplies have the least discriminant indices due to their similarity in topology and power consumption.

The design of an identification strategy based on the application of successive rules is encouraged for future works, by considering the indices with the best discrimination capacity according to this study.

\section{References}

[1] I. E. Agency, "Energy efficiency market report 2013," 2013. [2] P. Palensky and D. Dietrich, "Demand side management: Demand response, intelligent energy systems, and smart loads," Industrial Informatics, IEEE Transactions on, vol. 7, no. 3, pp. 381-388, Aug 2011.

[3] G. Hart, "Nonintrusive appliance load monitoring" Proceedings of the IEEE, vol. 80, no. 12, pp. 1870-1891, Dec 1992.

[4] EPRI, "Residential electricity use feedback: A research synthesis and economic framework," 2009.

[5] A. Zoha, A. Gluhak, M. Imran, and S. Rajasegarar, "NonIntrusive Load Monitoring Approaches for Disaggregated Energy Sensing: A Survey," Sensors, vol. 12, no. 12, pp. 16 838-16 866, Dec. 2012.

[6] A. Blanco, S. Yanchenko, J. Meyer, and P. Schegner, "Impact of supply voltage distortion on the harmonic emission of electronic household equipment," in SICEL, 2013.

[7]International standard PD IEC/TR 60725:2012. Consideration of reference impedances and public supply network impedances for use in determining disturbance characteristics of electrical equipment having a rated current $\leq$ 75 A per phase, Jul. 2012.

[8]Jimenez, Y.; Duarte, C.; Petit, J.; Carrillo, G., "Feature extraction for nonintrusive load monitoring based on STransform," Power Systems Conference (PSC), 2014 Clemson University, vol., no., pp.1,5, 11-14 March 2014 